\title{
EL DERECHO FUNDAMENTAL A UNA EDUCACIÓN \\ DE CALIDAD. ESPECIAL CONSIDERACIÓN A LA EDUCACIÓN SUPERIOR A DISTANCIA COMO ESTRATEGIA INSTITUCIONAL PARA POTENCIAR LA CALIDAD EDUCATIVA
}

\section{THE FUNDAMENTAL RIGHT TO A QUALITY EDUCATION. SPECIAL CONSIDERATION FOR HIGHER EDUCATION AS A CORPORATE STRATEGY TO ENHANCE THE QUALITY OF EDUCATION}

\author{
Juan Manuel Goig Martínez \\ UNED España \\ Valentina GobBo Coin \\ UNEG Venezuela
}

Resumen: La educación es un derecho fundamental vinculado a la dignidad y al libre desarrollo de la personalidad. Pero su contenido no se limita a cualquier proceso educativo, sino que para un correcto entendimiento del contenido del derecho a la educación, ésta tiene que ser una educación de calidad, que se mide por el cumplimiento de determinados parámetros. El modelo de educación superior a distancia, ofrece un modelo metodológico educativo adecuado como parámetro de calidad.

Abstract: Education is a fundamental right linked to the dignity and the free development of the personality. But its content is not limited to any educational process, but that this must be a quality education, which is measured by compliance with certain parameters for a correct understanding of the content of the right to 
education. Distance higher education model, offers a methodological educational model suitable as parameter of quality

Palabras clave: Derecho a la educación. Calidad educativa. Dignidad. Libre desarrollo de la personalidad. Educación superior a distancia.

Keywords: Right to education. Quality of education. Dignity. Free development of the personality. Distance higher education.

Recepción original: 04/09/2012

Aceptación original: 12/09/2012

Sumario: I. Dimensión internacional y contenido del derecho a la educación. La calidad educativa como contenido fundamental y posibilitador del derecho a la educación; II. La calidad educativa; II.1. Parámetros y dimensiones de la calidad de la educación; II.1.A. Respeto a la dignidad; II.1.B. La equidad; II.1.C. La igualdad y no discriminación; II.1.D. La inclusión; II.1.E. El acceso; II.1.F. La participación; II.1.G. La relevancia; II.1.H. La pertinencia; II.1.I. La eficacia; II.1.J. La eficiencia; II.2. Las actitudes de la Educación superior a distancia; III. Estrategias institucionales para potenciar la calidad. Concepción de la enseñanza universitaria como estrategia de calidad; III.1. La metodología de la educación superior a distancia como parámetro de calidad.

I. DIMENSIÓN INTERNACIONAL Y CONTENIDO DEL DERECHO A LA EDUCACIÓN. LA CALIDAD EDUCATIVA COMO CONTENIDO FUNDAMENTAL Y POSIBILITADOR DEL DERECHO A LA EDUCACIÓN

La educación es esencial para el desarrollo humano, y para el perfeccionamiento y la consolidación de la democracia.

Los sistemas educativos constituyen una pieza clave en el desarrollo de los Estados por cuanto recogen una serie de derechos fundamentales de los ciudadanos que deben de ser respetados y aplicados, y una serie de obligaciones que deben ser asumidas por los gobiernos.

El reconocimiento del derecho universal a la educación como derecho fundamental, es decir, un derecho irrenunciable y directamente exigible al que deben servir los poderes públicos y que debe constituir la directriz principal de las políticas públicas educativas, ya reconocido el artículo 13 de la Declaración Universal de Derechos Humanos de Naciones Unidas de 1948, pone de manifiesto la 
necesidad de hacer frente al principio de educación para todos y de igualdad de oportunidades y exige la necesidad de ampliar el acceso a la educación y el aprendizaje, liberando a los alumnos de las limitaciones de tiempo y espacio y ofreciendo oportunidades flexibles de aprendizaje a los individuos y a los grupos de alumnos.

Si el derecho a la educación es un derecho fundamental general, en tanto que «Todos tienen derecho a la educación», este derecho de «todos a la educación» constituye una obligación de garantía para los poderes públicos, lo que impone la necesaria intervención en aras a conseguir la efectividad de estos preceptos, y ello, no sólo derivará de su estrecha vinculación con la dignidad, sino por que la educación «tiene por objeto el pleno desarrollo de la personalidad humana», que, constituye uno de los fundamentos del orden político y de la paz social.

Esta consideración del derecho a la educación como un derecho fundamental, debe ser entendida como elemento imprescindible para asegurar que dicha educación se desarrolle en todas sus facetas, y ello, además, por la propia finalidad de este derecho: el libre desarrollo de la personalidad; la formación en el respeto de los derechos y libertades fundamentales y en el ejercicio de la tolerancia y la libertad dentro de los principios democráticos de convivencia, la preparación para participar activamente en la vida social y cultural y la formación para la paz, la cooperación y la solidaridad entre pueblos (art. 2 L.O.D.E; art. 1.1 LOGSE; art. 26.2 DUDH, y art. 13 PIDESC), determina, por un lado, que en el análisis de los derechos educativos han de estar permanentemente presentes los valores constitucionales de igualdad, libertad, justicia y pluralismo político; el propio concepto de Estado Social; y de otro, el arbitrar los mecanismos y modelos educativos precisos y adecuados para dotarlos de efectividad.

Organizaciones como la UNESCO continúan su lucha para que los países superen obstáculos e impartan una verdadera educación de calidad desde la primera infancia y a lo largo de toda la vida, entendiendo que la educación ayuda a combatir la pobreza, aumenta la producción económica, disminuye la delincuencia, por lo que redunda en beneficio de las naciones ${ }^{1}$.

En el Foro Mundial de Educación para Todos ${ }^{2}$ se estableció como uno de sus objetivos principales el alcanzar, para 2015, una educación para todos, es decir, que haya acceso, calidad y equidad.

\footnotetext{
${ }^{1}$ Conferencia Mundial sobre Educación para Todos. Declaración Mundial sobre Educación para Todos: la Satisfacción de las Necesidades Básicas de Aprendizaje. Jomtien, 1990

${ }^{2}$ Foro Mundial sobre educación. Educación para todos: cumplir nuestros com-
} 
Con ocasión del octavo centenario de la fundación de la Sorbona, el 25 de mayo de 1998 los Ministros de varias Universidades firmaban una «Declaración conjunta para la armonización del diseño del Sistema de Educación Superior Europeo» en el que se ponía de manifiesto la necesidad, de un proceso de cambios para las condiciones educativas y laborales, una diversificación del curso de las carreras profesionales, en el que la educación y la formación continua devienen una obligación evidente, porque debemos, tanto a los estudiantes, como a la sociedad en su conjunto, un sistema de educación superior que les ofrezca las mejores oportunidades para buscar y encontrar su propio ámbito de excelencia.

Desde 1998, con la Declaración de la Sorbona, en Europa se ha iniciado un proceso para promover la convergencia entre los sistemas nacionales de educación superior, que, con la Declaración de Bolonia de 1999, ha evidenciado la importancia de un desarrollo armónico de un Espacio Europeo de Educación Superior, a implantar antes de 2010, objetivo que está propiciando diversos foros de debate en todos los países miembros, para la evaluación de programas académicos convergentes que aseguren una calidad docente.

Estas necesidades armonizadoras también han tenido su correlato en la Declaración de Sao Paulo que ha surgido de la I Conferencia Interparlamentaria de Educación (2002), convocada por el PARLATINO y la UNESCO en la que se ha puesto de manifiesto que para lograr los objetivos de desarrollo e integración de los pueblos, es un requisito condicionante, esencial e impostergable, el asegurar una educación de calidad a todos, sin ningún tipo de condicionamiento, y a lo largo de toda la vida, y para el cumplimiento de estos objetivos es necesaria la armonización y la unidad funcional, para lo que se ha puesto en funcionamiento el Proyecto Regional de Educación de América latina y el Caribe, 2001-2015 (PREALC) y el Plan de Educación para el Desarrollo y la Integración de América Latina.

El ámbito internacional de los procesos educativos en la era de la globalización, implica la adopción de una serie de estrategias y principios, a lo que hay que unir, en lo que se refiere a los procesos educativos a distancia, una metodología común, que facilite la armonización.

Resulta evidente que una mejora de la calidad, entendida como aspiración de cualquier institución docente, exige una adecuación metodológica precisa, adecuación metodológica que, si es predicable

promisos comunes. Dakar, Senegal. 26 al 28 de Abril. Literal 1 (2000). 
de cualquier proceso formativo, adquiere una importancia especial en la educación universitaria a distancia por las especificidades propias de este modelo de enseñanza.

\section{LA CALIDAD EDUCATIVA}

\section{II.1. Parámetros y dimensiones de calidad educativa}

En el Informe de seguimiento de la Educación para Todos de la $\mathrm{UNESCO}^{3}$, se menciona que una educación de calidad debe abarcar tres dimensiones, que son fundamentales: la equidad, la pertinencia y la relevancia. Se considera, igualmente que deben añadirse otras de carácter operativo que son la eficacia y la eficiencia.

Sin éstas y otras dimensiones que son consideradas de importancia, no podría hablarse de educación de calidad: respeto a la dignidad, la equidad, la igualdad, la inclusión, el acceso, la participación, la relevancia, la pertinencia, la eficacia y la eficiencia.

\section{II.A. Respeto a la dignidad}

El respeto a la dignidad no es una dimensión en sí misma, pero se considera fundamental por cuanto el derecho a la educación de calidad es un derecho fundamental vinculado a ella.

Los derechos fundamentales se consideran como tales porque emanan de manera directa, como atributos que son esenciales, de la dignidad humana. Son facultades del individuo basados en la libertad, la igualdad y la seguridad del ser humano, como expresión de su dignidad.

Como indica González Pérez, la dignidad es el rango o categoría que corresponde al hombre como ser dotado de inteligencia y libertad, distinto y superior a todo lo creado, que comporta un tratamiento acorde, en todo momento, con la naturaleza humana ${ }^{4}$.

Debe considerarse a la educación como el más útil de los instrumentos al servicio de la libertad, que debe servir para satisfacer las aspiraciones y las necesidades de cada individuo, en cuanto a su formación se refiere, para alcanzar el pleno desarrollo de la perso-

${ }^{3}$ UNESCO. Educación para Todos. Ob. Cit.

4 González Pérez, J., La dignidad de la persona. Editorial Civitas. Madrid, 1986, p. 16.

(C) UNED. Revista de Derecho UNED, núm. 11, 2012 
nalidad, para que pueda explotar sus capacidades y aprovechar al máximo las oportunidades.

La educación debe orientarse hacia el pleno desarrollo de la personalidad humana y del sentido de su dignidad, y debe fortalecer el respeto por los derechos humanos y las libertades fundamentales. Estas líneas directrices están encaminadas a capacitar a todas las personas para participar efectivamente en una sociedad libre, favorecer la comprensión, la tolerancia y la amistad entre todas las naciones y entre todos los grupos raciales, étnicos o religiosos y promover el mantenimiento de la paz» ${ }^{5}$.

Para que el derecho a la educación de calidad sea tal, es necesario que ésta asegure que se respete el derecho a la propia identidad, esto es, respetar a cada persona tal y como es, con todas sus características, atendiendo de manera adecuada tanto el desarrollo de sus aptitudes como el respeto por sí mismo; atendiendo de igual forma sus necesidades individuales y darles, por ende, a cada alumno un trato justo que no atente contra su dignidad, permitiéndole desarrollar plenamente su personalidad y preparándolo para que logre alcanzar una vida personal, familiar y social digna.

\section{II.1.B. La equidad}

«Lo equitativo y lo justo son una misma cosa; y siendo buenos ambos, la única diferencia que hay entre ellos es que lo equitativo es mejor aún $»^{6}$.

El carácter universal que posee el derecho a la educación de calidad, por ser un derecho fundamental, hace necesario el observar si este derecho se hace efectivo para todos. La equidad consiste en aportar a cada quien los recursos y la ayuda que necesite para poder estar en igualdad de condiciones con los demás al momento de acceder a la educación en primer lugar, continuar con ella y adquirir los aprendizajes correspondientes a cada nivel educativo. La verdadera equidad es aquella que pasa por la democratización, a la hora de accesar al conocimiento y por la igualdad de oportunidades para que el individuo pueda desarrollar las competencias necesarias para participar en la sociedad y aumentar las posibilidades de cada uno a elegir.

${ }^{5}$ STS. Sala de lo Social. N. ${ }^{\circ}$ 1669/1994, del 19 de Septiembre de 1994.

${ }^{6}$ Aristóteles. Ética a Nicómaco, Libro V, Capítulo. X. Traducción de Mariano DE AzCÁrate. México, 1922. 
Establecer un criterio de equidad significa identificar cuál es la dimensión fundamental respecto a la cual definir un horizonte de igualdad y en torno a la cual se estructuran todas las desigualdades resultantes. Vista así, la equidad surge de las diferencias propias de los sujetos en los diversos contextos de la vida social. La equidad forma parte de la igualdad y es ampliada por sus dimensiones. El término equidad no acoge, al igual que la igualdad, la idea de que todos somos iguales, sino que parte del reconocimiento de la diversidad, y sugiere estrategias para alcanzar la igualdad necesaria que debería aportar la educación básica ${ }^{7}$.

Para que haya equidad, es necesario lograr una educación inclusiva y no excluyente ${ }^{8}$. de calidad, que pueda brindar oportunidades educativas a niveles de excelencia.

La equidad implica que haya igualdad de condiciones en el acceso: escuelas disponibles y accesibles a todos; igualmente debe haber equidad en los procesos, por lo que debe asegurarse el principio de igualdad de oportunidades; así mismo debe darse la equidad en los resultados de aprendizaje, es decir se deben alcanzar logros equiparables.

De igual modo, es necesario hablar de la equidad de género, es decir, se debe garantizar que tanto niñas como niños tengan acceso y logren permanecer en las aulas en proporciones similares. En la Declaración Mundial sobre Educación para Todos, se señala que «la prioridad más urgente es garantizar el acceso y mejorar la calidad de la educación para niñas y mujeres y suprimir todo obstáculo que impida su participación activa ${ }^{9}$. Como ya se había señalado es imprescindible que haya dentro de los centros educativos heterogeneidad de enfoques lo que contribuye a avanzar hacia una educación equitativa para hombres y mujeres de distintos grupos sociales y contextos, es decir, prepararlos para que puedan enfrentarse a las situaciones cotidianas, tener acceso a un empleo digno en igualdad de condiciones y oportunidades. Todos los niños y todas las niñas

${ }^{7}$ Calvo, G., La preocupación por la equidad y la formación docente. A propósito de la eficacia escolar. pág. 97-112. Blanco GuIJARRo, R. (Coordinadora). Eficacia Escolar y factores asociados en América Latina y El Caribe. UNESCO, Laboratorio Latino Americano de Evaluación de la Calidad de la Educación. Santiago de Chile. 2007, pág. 100.

${ }^{8}$ Blanco Guijarro, R., "La Educación de calidad para todos empieza en la primera infancia». UNESCO. Revista Enfoque Educacionales 7 (1):11-33, 2005. Departamento de Educación, Facultad de Ciencias Sociales, Universidad de Chile. Chile. p. 15 y sig.

${ }^{9}$ Conferencia Mundial sobre Educación para Todos. Ob. Cit., artículo III, Numeral 3. 
presentan diferentes capacidades y necesidades educativas por lo que deben ser atendidos de manera particular ${ }^{10}$.

Se puede deducir que la educación de «calidad y la equidad no solo no son incompatibles sino que son indisociables ${ }^{11}$. La educación es de calidad cuando ofrece todo lo necesario para que los alumnos alcancen sus niveles máximos de desarrollo y aprendizaje, de acuerdo con sus capacidades individuales; cuando todos los alumnos y no solo aquellos que pudieran llamarse privilegiados por las clases sociales o culturales a las que pertenecen, desarrollen las competencias que son necesarias para insertarse en la sociedad, tengan acceso a un empleo digno y puedan ejercer su libertad. Se está en presencia entonces de la equidad, vista desde una dimensión que resulta ser esencial, para así poder evaluar la calidad en la educación.

\section{II.1.C. La Igualdad}

Equidad no es lo mismo que igualdad, aunque están estrechamente vinculadas. La equidad abarca los principios de igualdad y diferenciación, ya que solamente una educación que logre ajustarse a las necesidades individuales de los alumnos, es la que asegura a todos las mismas oportunidades para hacer valer sus derechos y alcanzar la finalidad de la educación en condiciones de igualdad.

La igualdad admite una pluralidad conceptual que la configura como un valor, como un principio y como un derecho, y por lo tanto, se sitúa por encima de otros derechos que derivan de ella o que son manifestación de dicha igualdad. Como valor se sitúa en la cúspide de los enunciados constitucionales, puesto que informa todo el desarrollo constitucional.

En su condición de derecho, la igualdad constituye una facultad otorgada a la persona para proteger su posición y sus intereses, pero junto a esta manifestación subjetiva, el derecho fundamental a la igualdad implica obligaciones para los Poderes Públicos que, referidos a la materia educativa serán examinados más adelante.

La noción de igualdad deriva de la naturaleza del ser humano y es inseparable de la dignidad esencial de las personas frente a la cual es incompatible toda situación de discriminación, como ha indicado

${ }^{10}$ Blanco Guijarro, R., Ob. Cit. pág. 17.

${ }^{11}$ Educación de calidad para todos: Un asunto de Derechos Humanos. Ob. Cit. pág. 34. 
la Corte Interamericana de Derechos Humanos ${ }^{12}$ no es admisible crear diferencias de tratamiento entre seres humanos que no se correspondan con su única e idéntica naturaleza.

La igualdad consiste en "proporcionar más a quien más lo necesita y dar a cada uno las ayudas y recursos que requiere para que esté en igualdad de condiciones de aprovechar las oportunidades educativas ${ }^{13}$ ». La no discriminación implica tratar equitativamente a las personas que se encuentran en situación de igualdad y conceder un trato no igualitario a quienes se encuentran en una situación de desigualdad.

La Convención de la UNESCO relativa a la Lucha contra la Discriminación en la Esfera de la Enseñanza, entiende por discriminación toda distinción, exclusión, limitación o preferencia fundada en la raza, el color, el sexo, el idioma, la religión, las opiniones políticas o de cualquier otra índole, el origen nacional o social, la posición económica o el nacimiento que tenga por finalidad o por efecto destruir o alterar la igualdad de trato en la esfera de la enseñanza, y en especial: a) excluir a una persona o a un grupo del acceso a los diversos grados y tipos de enseñanza. b) limitar a un nivel inferior la educación de una persona o de un grupo. c) instituir o mantener sistemas o establecimientos de enseñanza separados para personas o grupos. d) colocar a una persona o a un grupo en una situación incompatible con la dignidad humana, y entiende que el término «enseñanza» se refiere a la educación en sus diversos tipos y grados y comprende el acceso a la enseñanza, el nivel y la calidad de ésta y las condiciones en que se $\mathrm{da}^{14}$.

Para que pueda ejercerse el derecho a la educación es requisito indispensable la no discriminación. Los principios que rigen a la educación han de ser los mismos para todos, independientemente de su origen y su condición socio-cultural, atendiendo las necesidades particulares de cada uno, por lo que se debe asegurar la igualdad de oportunidades ${ }^{15}$. Asegurar el derecho a una educación de calidad requiere, por lo tanto, asegurar el derecho a la no discriminación, por lo que todos, los niños y niñas deberían recibir educación juntos

${ }^{12}$ Corte Interamericana de Derechos Humanos. Opinión Consultiva N. ${ }^{\circ} 4$ del 19 de enero de 1984.

${ }^{13}$ Educación de Calidad para Todos: Un asunto de Derechos Humanos. Ob. Cit. pág. 35.

${ }^{14}$ UNESCO: Convención relativa a la Lucha contra la Discriminación en la Esfera de la Enseñanza. Art.1.

${ }^{15}$ Educación de Calidad para Todos: Un Asunto de Derechos Humanos. Ob. Cit. pág. 8.

(C) UNED. Revista de Derecho UNED, núm. 11, 2012 
en la escuela de su comunidad, sin importar su proveniencia, ni sus condiciones, ni sus características personales, para alcanzar el pleno desarrollo de su personalidad y aprender a vivir en medio de la diversidad y la tolerancia.

\section{II.1.D. La Inclusión}

La educación inclusiva «es la transformación de los sistemas educativos y de las escuelas comunes para dar respuesta a la diversidad del alumnado». La idea de la educación inclusiva es transformar las culturas, las prácticas educativas y la organización de las escuelas para atender las diversas necesidades en materia educativa de los estudiantes, necesidades derivadas de los aspectos sociales y culturales que rodean al alumno así como cada una de sus características personales como lo son la motivación, las capacidades, los intereses y las aptitudes.

La inclusión implica, no solo facilitar el acceso a las escuelas sino integrar en la diversidad, lo que significa que la educación debe estar basada en la diversidad y no en la homogeneidad. Cuando se habla de diversidad o diferencia entre los estudiantes no debe confundirse con desigualdad, ya que la diversidad implica el aseguramiento del derecho a la propia identidad

La inclusión debe ser un proceso que vaya dirigido a responder a las distintas necesidades de los estudiantes, que logre incrementar la participación de los mismos en el aprendizaje, las culturas y las comunidades, por lo que es necesario que en las escuelas se dé una profunda transformación ya que deben adaptarse a las diferencias de aprendizajes de los alumnos y no los alumnos adaptarse a lo que está disponible en la oferta académica, esto conllevaría a la reducción de la exclusión en y desde la educación.

\section{II.1.E. El Acceso}

El acceso es una condición necesaria para que pueda ejercerse plenamente el derecho a la educación, pero no es suficiente, ya que se requieren de todas las demás dimensiones. El acceso consiste en lograr matricularse, asistir a la escuela y permanecer durante todo el proceso de enseñanza-aprendizaje. 
El acceso abarca elementos como la accesibilidad física, accesibilidad al currículo y accesibilidad económica ${ }^{16}$. Es el Estado quien tiene la responsabilidad de garantizar el derecho a la escolarización, tienen el deber de asegurarles a los niños y sus familias el acceso universal a las escuelas, así como la prosecución y la culminación de los distintos niveles educativos.

\section{II.1.F. La Participación}

Definimos participación como la intervención de un individuo o grupo organizado en la toma de decisiones, que busca, por un lado, permitir que quien toma la decisión definitiva esté informado de nuestra opinión y, por otro, colaborar para que ésta se haga realidad, fiscalizar que ésta se haga realidad apropiadamente o cambiar la decisión en caso de no estar de acuerdo ${ }^{17}$.

Las escuelas deben ser el núcleo que llame a las comunidades locales a participar, para alcanzar la mejora de la calidad educativa, partiendo desde el principio en que ambos (escuela y comunidad), deben tener claro los propósitos que los une, lograr la excelencia académica.

Pero la participación no solo consiste en tomar parte de las actividades programadas, es también tener la posibilidad de tomar, de manera compartida, las decisiones que afecten la propia vida y de la comunidad donde vivan los estudiantes y sus padres o representantes. También es el derecho a expresar opiniones, lo cual es necesario para ejercer plenamente los procesos democráticos requeridos dentro de las comunidades ya que esto hace a los padres, representantes, docentes de los centros educativos y al estudiantado, protagonistas y responsables de la educación de los niños de dicha comunidad, además esto permite una mayor transparencia por tener el control de las decisiones y de los resultados de las mismas ${ }^{18}$.

La participación de la comunidad en la escuela pudiera generar aportes que se traduzcan en valores como son la solidaridad, la coo-

${ }^{16}$ Educación de Calidad para Todos: Un asunto de Derechos Humanos. Ob. Cit. pág. 36.

${ }^{17}$ Serrano, A., "De la Participación Entendida como Exclusión. Una Reflexión sobre la Asamblea de Ciudadanos". Revista Guayana Sustentable 5. 2004 Universidad Católica Andrés Bello. Fundación Konrad Adenauer Stift6ung. Caracas. págs. 83-92. pág. 85.

${ }^{18}$ Educación de Calidad de Todos: Un Asunto de Derechos Humanos. Ob. Cit. pág. 33. 
peración, nuevas formas de convivencia y relaciones sociales. Esto conllevaría que los actores inmersos en la participación aporten iniciativas, ideas, sugerencias basadas en su experiencia, que se transformen en acciones que beneficien a la escuela. Alcanzar la participación de la ciudadanía en la educación es un reto para la sociedad, desde allí podrían vencerse obstáculos como la inclusión, el acceso, la discriminación, entre otros.

\section{II.1.G. La Relevancia}

La educación será relevante en la medida que promueva aprendizajes significativos desde el punto de vista de las exigencias sociales y de desarrollo personal, lo cual difícilmente ocurrirá si ésta no es también pertinente, es decir si no considera las diferencias para aprender que son el fruto de las características y necesidades de cada persona, las cuales están a su vez mediatizadas por el contexto social y cultural en que viven ${ }^{19}$.

No hay educación de calidad si los contenidos que se imparten en las escuelas no son relevantes, tanto para alcanzar el desarrollo de las Naciones como, aquellos necesarios para lograr el desarrollo pleno de los estudiantes. La formación que deben ofrecer las escuelas ha de capacitar a los alumnos para que participen con responsabilidad en los diversos ámbitos de la vida humana, para que puedan afrontar los desafíos de la sociedad y para que puedan planificar y construir sus proyectos de vida acordes con las necesidades personales y del país ${ }^{20}$.

Relevancia está relacionada con los sentidos de la educación, sus finalidades y contenido, y con el grado en que ésta satisface efectivamente las necesidades, aspiraciones e intereses del conjunto de la sociedad y no solamente de los grupos con mayor poder dentro de la misma. Desde la perspectiva del derecho internacional, a la educación se le atribuyen cuatro finalidades fundamentales: lograr el pleno desarrollo de la personalidad y de la dignidad humana, fomentar el respeto de los derechos y libertades fundamentales, fomentar la participación en una sociedad libre, y fomentar la comprensión, la

${ }^{19}$ Vid. Maldonado P. R., «Relevancia y Pertinencia en la Educación». Revista Digital Huellas Digitales. 2007, Chile.

${ }^{20}$ Blanco Guijarro, R., "La calidad de la Educación: Un asunto de Derechos Humanos». Ob. Cit. pág. 2. 
tolerancia y las relaciones entre todas las naciones, grupos religiosos o raciales, y el mantenimiento de la paz ${ }^{21}$.

Desarrollar de manera plena e integral la personalidad humana, es una de las finalidades que debe tener la educación. Por lo que la educación de calidad es relevante, si se encuentra orientada a cumplir las finalidades que son fundamentales en un momento dado, dentro de las sociedades ${ }^{22}$, es importante además que ésta se vaya adecuando al mundo de hoy, que es un mundo cambiante y en continuo movimiento. La relevancia es el qué y el para qué de la educación.

Se entiende que la educación es relevante si logra promover el aprendizaje de las competencias necesarias para participar en la sociedad, para afrontar los desafíos que ésta les imponga, acceder a un empleo digno, desarrollar los proyectos de vida, vistos tanto de manera individual como social. Desde este punto de vista la UNESCO considera que para el siglo XXI el estudiante debería desarrollar competencias relacionadas con "aprender a conocer, aprender a hacer, aprender a ser y aprender a vivir juntos.»

\section{II.1.H. La Pertinencia}

La pertinencia de la educación se refiere a la necesidad de que sea significativa para todas las personas, ya sean éstas de distintos estratos socio-culturales y con diferentes capacidades e intereses, de forma tal, que puedan apropiarse de los contenidos referentes a la cultura mundial y local y formarse como personas, buscando el pleno desarrollo de su autonomía y de su propia identidad.

Para que la educación sea pertinente, debe ser flexible y debe lograr adaptarse a las necesidades individuales de los estudiantes y de sus contextos, tanto sociales como culturales. Significa entonces, que la educación ha de transitar por la heterogeneidad aprovechando a ésta como una oportunidad para enriquecer los procesos educativos y permitir al alumno el desarrollar el ser, como persona y como ente de la sociedad.

La educación es pertinente si considera al estudiante como su eje central, por lo que debe adecuar el proceso de enseñanza-aprendizaje a sus necesidades y características particulares, partiendo de

${ }^{21}$ Blanco Guijarro, R. (Coordinadora). "Eficacia Escolar y factores asociados en América Latina y El Caribe». UNESCO, Laboratorio Latino americano de Evaluación de la Calidad de la Educación. Santiago de Chile. 2007, pág. 12.

22 "Educación de Calidad de Todos: Un Asunto de Derechos Humanos». Ob. Cit. pág. 9.

(C) UNED. Revista de Derecho UNED, núm. 11, 2012 
lo que «es», «sabe»y «siente», visto desde su contexto socio-cultural y buscando promover el desarrollo de sus diferentes capacidades e intereses. Se entiende entonces, que es necesario que haya una transformación profunda de las prácticas educativas, educando desde la diversidad, aprovechándola como oportunidad enriquecedora de los procesos educativos, lo cual permitiría alzar los valores contra la discriminación que cada día acechan a los estudiantes y así permitiría que el alumno alcance el pleno desarrollo de su personalidad.

\section{II.1.I. La Eficacia}

La eficacia consiste en analizar en qué medida se logran alcanzar las metas, así como los aspectos concretos y necesarios para que se cumpla el derecho a una educación de calidad para todos ${ }^{23}$. Una escuela eficaz es aquella que consigue un desarrollo integral de todos y cada uno de sus alumnos, mayor de lo que sería esperable teniendo en cuenta su rendimiento previo y la situación social, económica y cultural de las familias ${ }^{24}$.

Las metas, que al menos, se deben alcanzar son las establecidas en el informe de Educación para Todos de Dakar ${ }^{25}$ :

a) Atención a la primera infancia, dado, como ya se ha mencionado, que comenzar a temprana edad trae mejores y mayores beneficios para el alumno.

b) Que todos los estudiantes logren concluir la educación primaria.

c) La necesidad de que sea atendida la educación para adultos, para lograr así la reducción del analfabetismo entre éstos.

d) Que haya equidad de género, no solo en el acceso sino también en las oportunidades.

e) Que se alcance tener una educación de calidad para todos.

Un sistema de educación demuestra su eficacia cuando es capaz de cumplir su objetivo esencial: dotar a los jóvenes con las competencias que necesitan para encontrar medios seguros de subsistencia y participar activamente en la vida social, económica y política de sus comunidades ${ }^{26}$.

${ }^{23}$ Blanco Guijarro, R., Ob. Cit. pág. 10 y sigs.

${ }^{24}$ Murillo, F. J. (2005). «La investigación sobre Eficacia Escolar». Ediciones Octaedro. Barcelona, pág. 25

${ }^{25}$ Foro Mundial sobre educación. Ob. Cit.

${ }^{26}$ UNESCO. (2011). "Una Crisis Encubierta: Conflictos Armados y Educación.» Informe de seguimiento de la Educación para Todos en el mundo 2011. Ediciones UNESCO. París, Francia. pág. 19. 


\section{II.1.J. La Eficiencia}

La eficiencia implica, analizar en qué medida el Estado asigna los recursos que son necesarios y si éstos se utilizan y distribuyen de forma adecuada para poder lograr una educación de calidad para todos, es decir, que sea equitativa, relevante y pertinente ${ }^{27}$.

No significa necesariamente, que el Estado deba gastar más en educación sino que debe gastar mejor, de manera planificada para maximizar la cobertura, es necesario que los recursos que se asignan a la educación, a las escuelas permitan alcanzar los fines establecidos y así poder lograr la calidad en la educación. La distribución debe hacerse de tal forma que no genere desigualdad y se mantenga la equidad.

\section{II.2. Las actitudes de la Educación superior a distancia}

Como hemos indicado, las sociedades actuales conceden gran importancia a la educación que reciben sus jóvenes, en la convicción de que de ella dependen tanto el bienestar individual como el colectivo. La educación es el medio más adecuado para construir su personalidad, desarrollar al máximo sus capacidades, conformar su propia identidad personal y configurar su comprensión de la realidad, integrando la dimensión cognoscitiva, la afectiva y la axiológica. Para la sociedad, la educación es el medio de transmitir y, al mismo tiempo, de renovar la cultura y el acervo de conocimientos y valores que la sustentan, de extraer las máximas posibilidades de sus fuentes de riqueza, de fomentar la convivencia democrática y el respeto a las diferencias individuales, de promover la solidaridad y evitar la discriminación, con el objetivo fundamental de lograr la necesaria cohesión social. Además, la educación es el medio más adecuado para garantizar el ejercicio de la ciudadanía democrática, responsable, libre y crítica, que resulta indispensable para la constitución de sociedades avanzadas, dinámicas y justas. Por ese motivo, una buena educación es la mayor riqueza y el principal recurso de un país y de sus ciudadanos.

La concepción de la educación como un instrumento de mejora de la condición humana y de la vida colectiva ha sido una constante, aunque no siempre esa aspiración se haya convertido en realidad. Ahora bien, en cada fase de su evolución, los sistemas educativos

${ }^{27}$ Blanco Guijarro, R. Ob. Cit. pág. 10 y sigs. 
han tenido que responder a unos retos prioritarios, y desde los años finales del siglo XX, el desafío consistió en conseguir que esa educación ampliamente generalizada fuese ofrecida en unas condiciones de alta calidad, con la exigencia además de que tal beneficio alcanzase a todos los ciudadanos. Ello ha llevado a los Estados a buscar parámetros y compromisos para hacer de la calidad educativa un elemento necesario y definidor del derecho a la educación.

Lograr que todos los ciudadanos puedan recibir una educación y una formación de calidad, sin que ese bien quede limitado solamente a algunas personas o sectores sociales, resulta acuciante en el momento actual. Países muy diversos, con sistemas políticos distintos y gobiernos de diferente orientación, se están planteando ese objetivo.

Tres son los principios que debe de cumplir todo modelo educativo:

- Proporcionar una educación de calidad a todos los ciudadanos y en todos los niveles educativos;

- La necesidad de que todos los componentes de la comunidad educativa colaboren para conseguir ese objetivo; y

- Un compromiso Internacional con los objetivos educativos planteados.

La Comunidad Internacional se ha propuesto mejorar la calidad y la eficacia de los sistemas educativos y de formación, lo que implica mejorar la capacitación de los docentes, desarrollar las aptitudes necesarias para la sociedad del conocimiento, garantizar el acceso de todos a las tecnologías de la información y la comunicación, aumentar la matriculación en los estudios científicos, técnicos y artísticos y aprovechar al máximo los recursos disponibles, aumentando la inversión en recursos humanos. En segundo lugar, se ha planteado facilitar el acceso generalizado a los sistemas de educación y formación, lo que supone construir un entorno de aprendizaje abierto, hacer el aprendizaje más atractivo y promocionar la ciudadanía activa, la igualdad de oportunidades y la cohesión social. En tercer lugar, se ha marcado el objetivo de abrir estos sistemas al mundo exterior.

Para conseguir que estos principios se conviertan en realidad, hay que actuar en varias direcciones complementarias:

- En primer lugar, se debe concebir la formación como un proceso permanente, que se desarrolla durante toda la vida.

- En segundo lugar, la existencia de un marco legislativo capaz de combinar objetivos y normas comunes con la necesaria autonomía pedagógica y de gestión de los centros docentes, lo 
que obliga, por otra parte, a establecer mecanismos de evaluación y de rendición de cuentas.

- En tercer lugar, es necesario incrementar la flexibilidad del sistema educativo.

Pues bien, para la consecución de estos fines, la educación a distancia debe de estar sobradamente preparada, puesto que su metodología ofrece respuestas a los objetivos marcados.

Si reconocemos que las Universidades ocupan un papel central en el desarrollo cultural, económico y social de un país, será necesario reforzar su capacidad de liderazgo y dotar a sus estructuras de la mayor flexibilidad para afrontar estrategias diferenciadas en el marco de un escenario vertebrado. Esta capacidad les permitirá desarrollar a cada una de ellas planes específicos acordes con sus características propias, con la composición de su profesorado, su oferta de estudios y con sus procesos de gestión e innovación. Sólo así, la sociedad podrá exigir de sus Universidades la más valiosa de las herencias para su futuro: una docencia de calidad, una investigación de excelencia.

En el orden Internacional, la necesidad de potenciar y desarrollar la metodología de enseñanza a distancia se ha puesto de manifiesto en numerosos Instrumentos ${ }^{28}$.

A este objeto, la propia Unión Europea, a través del Consejo de Ministros de Educación, en reunión celebrada el 27 de noviembre de 1992, y con el fin de establecer una serie de criterios para el fomento de la política educativa comunitaria en el ámbito de la enseñanza a distancia, se pone de manifiesto la necesidad de facilitar la cooperación entre instituciones y organismos del sector, incrementar la formación de formadores; resaltar la importancia de la calidad y necesidad de tutorías adecuadas; y la necesaria consulta a los sectores y usuarios

28

- La recomendación 1110 de 6 de julio de 1989 de la Asamblea del Consejo de Europa manifiesta la necesidad de fomentar la cooperación intergubernamental en el ámbito de la educación a distancia.

- El Informe sobre la Enseñanza Superior a Distancia en la Comunidad Europea de 1991, emanado del Parlamento Europeo indica la necesidad de la formación contínua para el desarrollo y progreso personal y social y la adecuación del modelo de educación a distancia para el desarrollo de estas políticas educativas.

- El artículo 126 del Tratado de Maastrich recomienda la potenciación y estímulo de la educación a distancia para fomentar las acciones prioritarias de la UE en el ámbito de la educación, formación profesional y juventud.

- El artículo 13 de la Carta de los Derechos Fundamentales de la Unión Europea (DOCE C364 de 18 de diciembre de 2001), al reconocer el derecho de toda persona a la educación, lo extiende al acceso a la formación permanente. 
inmersos en este modelo educativo como punto de partida para el conocimiento de las excelencias y deficiencias que hayan podido ser observadas $^{29}$.

No obstante, sin duda, el mayor impulso en la utilización del aprendizaje a distancia como mecanismo para fomentar la educación permanente, y de calidad, para todos, ha venido de la mano de la UNESCO, que para favorecer el desarrollo de esta estrategia, ha manifestado en la Estrategia a Medio Plazo para el período 1996-2001 las siguientes necesidades:

- La renovación y diversificación de los sistemas educativos en su conjunto; y la expansión y diversificación de las estructuras, al objeto de desarrollar las oportunidades educativas alternativas y sistemas de difusión, tanto en la enseñanza formal como en la no formal, haciendo un llamamiento a un uso más amplio de la educación a distancia.

- El desarrollo de un mayor uso del aprendizaje a distancia para aumentar el acceso a la educación y mejorar su efectividad en todas las formas, tipos y niveles, incluyendo el potencial de los sistemas educativos de difusión, utilizando las más modernas tecnologías de información y comunicación.

- La necesidad de formar, actualizar y motivar a los profesores y otros agentes sociales, utilizando enfoques innovadores que incluyan la educación a distancia.

La educación superior a distancia, y ponemos nuestro énfasis en el modelo de educación universitaria puesto que es en el que nos encontramos incardinados, aunque perfectamente podría aplicarse a los distintos niveles educativos, y la UNED como institución prototipo de este modelo educativo en el Estado español, da cumplimiento a la necesidad de democratizar el acceso a la educación, propiciando un aprendizaje autónomo y ligado a la experiencia en el que se capacita al alumno, primero en la idea de «aprender a aprender» y a «aprender a tecnificarse» aprovechando sus condiciones y autonomía en lo que respecta a tiempo, estilo, ritmos y método de aprendizaje, y fomentando la educación permanente impartiendo una enseñanza innovadora, pero que mantenga una calidad que la sitúe, sino por encima, al menos en paridad de rango respecto a los modelos tradicionales de enseñanza.

Si los objetivos de la democratización y la autonomía han sido perseguidos durante décadas por la Universidad, y hoy ya están razo-

${ }^{29}$ IUED. Aprendizaje abierto y a distancia... Op. cit, págs 25 y 26. 
nablemente alcanzados en España, en la actualidad los objetivos que preocupan en el ámbito universitario son los referidos a la calidad, la eficacia y la eficiencia.

Una preocupación constante de las Universidades, y entre ellas la UNED, ha sido la de mejorar sus procesos y resultados académicos e investigadores, puesto que la búsqueda y consecución de la excelencia académica y científica es una vocación irrenunciable de las instituciones de educación superior que las empuja a esforzarse continuamente en la mejora de los servicios que prestan a la sociedad en el campo de la formación, la enseñanza, la investigación y la cultura.

Resulta evidente que una mejora de la calidad, entendida como aspiración de cualquier institución docente, exige una adecuación metodológica precisa, adecuación metodológica que, si es predicable de cualquier proceso formativo, adquiere una importancia especial en la educación universitaria a distancia por las especificidades propias de este modelo de enseñanza.

Estudiar la metodología de la UNED como Universidad a Distancia de ámbito nacional, nos exige, por un lado el reconocimiento y conocimiento de las virtudes y desventajas que el modelo de enseñanza universitaria posee respecto al modelo «presencial», y por otro, marcar los perfiles adecuados de los elementos que integran este proceso metodológico.

En lo que al primer punto se refiere-excelencias y deficiencias-de la educación superior a distancia ${ }^{30}$, la propia Universidad y el profesor que ejerce, o aspira a ejercer, su actividad docente e investigadora en este ámbito, debe de conocer que la educación a distancia constituye una modalidad educativa que es capaz de dar respuesta eficaz a multitud de necesidades educativas.

Pero se trata, además de que la UNED y los sectores en ella implicados sean conscientes de las ventajas que el sistema implica respecto a la apertura, la eficacia, la economía, la flexibilidad y la formación permanente y personal, que seamos capaces de entender, al objeto de intentar superarlos, algunos inconvenientes que pueden ser inherentes al modelo educativo, entre los que se encuentran:

1. las dificultades que pueden surgir para orientar la actitud de los alumnos.

${ }^{30}$ Un profundo estudio sobre las ventajas y desventajas del modelo educativo de la UNED puede verse en GARCÍA AREITO, L., «Respondiendo a los tópicos, recelos, resistencias y temores» en Boletín Informativo de la Asociación iberoamericana de Educación Superior a Distancia, n. ${ }^{\circ}$ 14, 1986. 
2. la necesaria anticipación y la adecuada planificación de las actividades docentes.

3. las escasas ocasiones en que se puede ejercer la relación directa educativa entre profesor y alumno, con lo que ello tiene de inconveniente.

4. la necesidad de formar a los propios docentes en la metodología a distancia a fin de acortar la lejanía respecto a sus alumnos.

5. la adecuada organización administrativa y gestora que es mucho más compleja que en la educación presencial

6. las dificultades para evitar el mayor abandono o la sensación de fracaso entre los alumnos.

7. el establecimiento de técnicas adecuadas para la evaluación del aprendizaje en alumnos que, generalmente, son desconocidos para el docente, y sobre el que es imposible la aplicación de una evaluación continua que pudiera evitar la realización de un examen.

\section{ESTRATEGIAS INSTITUCIONALES PARA POTENCIAR LA CALIDAD. CONCEPCIÓN DE LA ENSEÑANZA UNIVERSITARIA COMO ESTRATEGIA DE CALIDAD}

La fijación de un método docente adecuado a los fines que consideramos debe obedecer nuestra labor formativa y educativa, debe de partir de la observación de dos realidades básicas: lo que se considere que son los fines de la Universidad, y la situación en que ésta se encuentra en el momento de la aplicación de una metodología concreta.

La legislación orgánica en materia universitaria ha reconocido que la Universidad realiza el servicio público de la educación superior mediante la investigación, la docencia y el estudio, siendo funciones de la Universidad al servicio de la sociedad:

a) La creación, desarrollo y trasmisión y crítica de la ciencia, de la técnica y de la cultura.

b) La preparación para el ejercicio de actividades profesionales que exijan la aplicación de conocimientos y métodos científicos y para la creación artística.

c) La difusión, la valoración y la trasferencia del conocimiento al servicio de la cultura, de la calidad de la vida y del desarrollo económico.

d) La difusión del conocimiento y la cultura a través de la extensión universitaria la formación a lo largo de toda la vida. 
Esta descripción legal de lo que debe ser entendida la misión de la Universidad, tiene, sin embargo, que ser considerada en su conjunto, de forma que no podamos detenernos en uno sólo de sus contenidos. La Universidad debe desarrollar un quehacer que vaya más allá de la mera capacitación profesional, y el Profesor debe ser consciente de cual es su misión: educar, esto es, extraer de dentro hacia afuera, de forma que la labor docente no debe orientarse exclusivamente a una trasmisión aséptica de determinada información, sino que debe favorecer la trasmisión de la ciencia, pero también el desarrollo, crítica y la creación de la misma.

La Universidad está para hacer ciencia y teorizar, favoreciendo la investigación para saber, pero también induciendo el saber para enseñar, y además, en un mundo que está cambiando de forma vertiginosa, la Universidad deberá de permitir el análisis científico de los hechos indicadores de ese cambio, siendo permeable a los problemas de la sociedad, colaborando en la búsqueda de soluciones ${ }^{31}$.

Creemos que la enseñanza universitaria debe ser, en primer lugar, la enseñanza de la necesidad de una ciencia y esta enseñanza debe favorecer el tránsito de la «idea creencia» a la «idea concurrencia», de forma que la duda como fallo en la creencia sea superada por la idea ${ }^{32}$. El docente debe crear, o al menos intentarlo, una mentalidad crítica, debe enseñar a pensar con rigor, a distinguir lo verdadero de lo falso, a dominar el mecanismo de la justificación, a entender, de tal manera que cuando no se entienda se sepa que no se entiende, de modo que cuestione los temas que le lleguen, pero de acuerdo con un método lógico y sistemático.

De esta forma se supera la idea de la Universidad como preparación o educación para la vida profesional y para el trabajo, que también es misión de la Universidad, y se hace nuestra la idea de que la Universidad también debe educar para la libertad personal y preparar las disponibilidades precisas para permitir la adaptación al cambio.

$\mathrm{Y}$ en este sentido, la tarea del docente no solamente debe ir orientada a manifestar creencias y realidades, sino que también debe señalar contradicciones y generar algunas inseguridades, con el fin de despertar inquietudes y vocaciones y, en la medida de lo posible, canalizarlas, aportando los medios precisos para que el universitario pueda reali-

${ }^{31}$ González Navaro, F., «La Universidad en la que yo creo» en $R A P$, n. ${ }^{\circ}$ 153, 2000, págs. 107 y ss.

32 GonZález Navarro, F., Derecho Administrativo español, Pamplona, EUNSA, 1993, págs 598 y ss. 
zarse desde el punto de vista intelectual y desde el punto de vista personal, para lo que precisa no sólo de una sólida formación científica, sino de un Método docente adecuado. El Profesor debe desvelar unas actitudes, suscitando en el alumno una inquietud intelectual, haciéndole ver la necesidad y la utilidad del uso de un método, favoreciendo un aprendizaje innovador ${ }^{33}$.

Ahora bien, visto ésto, también es cierto que el docente debe hacer frente a la situación concreta a la que se enfrenta en su quehacer diario. Y esta situación, en la actualidad, viene marcada por dos características: la situación de la Universidad y la predisposición del alumno.

La situación de la institución universitaria se ha trasformado de forma radical. Evidentemente, el reconocimiento, al menos teórico, de la igualdad de todos respecto de la educación y del correlativo aumento de las expectativas educativas, ha favorecido una Universidad de masas en la que el docente debe ejercer su función educativa, lo que dificulta un contacto más directo y personal que, con toda seguridad, es el deseado por el Profesor Universitario. Pero además, nos vemos inmersos en otros problemas que afectan a la Universidad, y que determinan que la labor docente encuentre serias dificultades en el ejercicio de la actividad de creación científica y dedique su actividad pedagógica a enseñar la creación científica, pero no tanto a inyectarla y digerirla ${ }^{34}$.

La otra cuestión que debe ser conocida para que el docente adapte su método a la realidad en que se desenvuelve es la predisposición del alumnado que inicia su formación universitaria.

Aunque no es este el deseo del Profesor Universitario, la realidad práctica es que la mayoría de los alumnos no vienen a aprender a hacer ciencia, sino a aprender una ciencia ya inventada, y ya creada, para aplicarla en su futuro profesional, realidad que debe ser tenida en cuenta por el docente. Se trata, como afirmaba Ortega ${ }^{35}$, de saber separar la enseñanza para el ejercicio profesional de la investigación científica, tarea ésta que exigirá del docente la aportación de importantes dosis de contenido y conocimientos.

Sin embargo esta apreciación y conocimiento de la realidad no debe llevar al docente al desánimo y a renunciar a inspirar ciertos va-

${ }^{33}$ FonT y Llovet, T., «Enseñanza, aprendizaje y educación en el Derecho Administrativo» en $R A P, \mathrm{n}^{\circ} 153,2000$, págs. 254 y ss.

${ }^{34}$ Ortega y Gasset, J., «Misión de la Universidad», 3. a ed. Revista de Occidente, Madrid, 1960, pág 39.

35 Ortega y Gasset, J., Op. Cit, págs 40 y ss. 
lores e intentar despertar ciertas actitudes y aptitudes en sus alumnos, sino que, por el contrario, exigirá del docente la capacidad de intentar distinguir en su alumnado entre aquellos que aspiran a algo más que a la mera adquisición de conocimientos y aquellos otros que vienen con una mentalidad predispuesta.

\section{II.1. La metodología de la educación superior a distancia como parámetro de calidad}

A pesar de que han sido muy numerosas las definiciones referentes a la educación a distancia ${ }^{36}$, que parten de una diversidad en la consideración de los proyectos, el tamaño, las estructuras, la organización o incluso, la denominación, nos alineamos con la definición de FERNANDEZ DE BUJAN, para quien «la educación a distancia es un sistema de enseñanza apto para ser ofrecido a un amplio número de estudiantes, en el que los alumnos, sin requerir la presencia continua de sus profesores, y con el fin de cursar de forma flexible sus estudios, dirigen personalmente su propio proceso de aprendizaje, aprovechándose de los medios tecnológicos y el apoyo tutorial que les ofrece un equipo docente, responsable de la programación académica, la elaboración de los materiales didácticos y la evaluación de los resultados» ${ }^{37}$.

De la consideración de la enseñanza a distancia como «un sistema tecnológico de comunicación bidireccional, que puede ser masivo y que sustituye la interacción personal en el aula de profesores y alumnos como medio preferente de enseñanza por la acción sistemática y conjunta de diversos recursos didácticos y el apoyo de una organización y tutoría que propician el aprendizaje independiente y flexible de los estudiantes ${ }^{38}$, podemos extraer una serie de características, cuya combinación, y mayor o menor ponderación, concurren en la consideración de la enseñanza a distancia:

1. En la modalidad de educación a distancia existe una importante separación profesor-alumno, lo que no implica una ausencia de relación entre los alumnos y los docentes.

2. Se establece una organización de apoyo al alumno a través de un sistema tutorial.

${ }^{36}$ Una completa exposición de las diversas definiciones sobre la enseñanza a distancia pueden verse en GARCÍA AREITO, L. (Coord). La Educación a Distancia y la UNED, UNED-IUED, Madrid, 1996, págs 35 y ss.

${ }^{37}$ FERNÁNDEZ DE BuJÁN, F., La enseñanza universitaria a distancia. Una reflexión desde la UNED. UNED, Madrid, 2001, pág 286.

${ }^{38}$ García Areito, L. (Coord). Op. cit, pág 50. 
3. En la modalidad de la enseñanza a distancia se potencia el aprendizaje individual y flexible y se favorece el trabajo independiente del alumno. No obstante este aprendizaje independiente y flexible no significa que el alumno se encuentre sin los medios de apoyo necesarios para solventar cualquier problema que se pudiera suscitar. Por el contrario, si el modelo de educación a distancia dispone de medios necesarios y diversos para ofrecer soluciones a estos problemas, lo que se pretende potenciar es que el alumno adecue estos medios, y opte entre ellos, a sus propias necesidades.

4. En la educación a distancia existe una comunicación bidireccional en el que ambas partes (docentes y alumno) se convierten en emisores y receptores.

La enseñanza a distancia constituye, además, un factor innovador de los sistemas educativos y este carácter innovador se manifiesta por la flexibilidad para facilitar cualquier tipo de aprendizaje y responder a las demandas más variadas;. Se trata, además, de innovaciones que están constituyendo un reto para la renovación de los «sistemas tradicionales» de educación.

El reconocimiento del derecho universal a la educación como derecho fundamental, es decir, un derecho irrenunciable y directamente exigible al que deben servir los poderes públicos y que debe constituir la directriz principal de las políticas públicas educativas, ya reconocido el artículo 13 de la Declaración Universal de Derechos Humanos de Naciones Unidas de 1948, pone de manifiesto la necesidad de hacer frente al principio de «educación para todos y de igualdad de oportunidades y exige la necesidad de ampliar el acceso a la educación y el aprendizaje, liberando a los alumnos de las limitaciones de tiempo y espacio y ofreciendo oportunidades flexibles de aprendizaje a los individuos y a los grupos de alumnos».

De esta forma, las Universidades a Distancia están caracterizadas por todos y cada uno de los rasgos generales y propios de la Universidad: autonomía académica y económica; personalidad jurídica; plenas funciones docentes e investigadoras y capacidad para otorgar títulos universitarios del mismo rango oficial que los de las otras Universidades. Pero, a la vez, son Universidades con características singulares que la diferencian de las otras en lo que hace referencia a: sus destinatarios más comunes; circunscripción geográfica; estructura territorial; medios utilizados en la relación docente-discente; una de su tipología de profesores (los Profesores Tutores) y sus funciones. 
Las medidas encaminadas a promover la mejora de la calidad del sistema educativo, se deberían organizar en torno a cuatro ejes fundamentales:

- En primer lugar, en la convicción de que los valores del esfuerzo y de la exigencia personal constituyen condiciones básicas para la mejora de la calidad del sistema educativo.

- El segundo eje de medidas, consiste en orientar más abiertamente el sistema educativo hacia los resultados.

- El tercero de los ejes, consiste en reforzar significativamente un sistema de oportunidades de calidad para todos.

- El cuarto eje se refiere al profesorado. Por la fundamental importancia que tiene la calidad de la relación profesor-alumno, núcleo de la educación, para obtener buenos resultados escolares, y por el elevado efecto multiplicador que dicha relación comporta, las políticas dirigidas al profesorado constituyen el elemento más valioso y decisivo a la hora de lograr la eficacia y la eficiencia de los sistemas de educación y de formación.

La mejora de la calidad del sistema universitario en su conjunto y en todas y cada una de sus vertientes, se constituye en elemento irrenunciable, y ello exige el establecimiento de nuevos mecanismos para el fomento de la excelencia, al objeto de:

- Mejorar la calidad de la gestión, mediante procedimientos que permitirán resolver con agilidad y eficacia las cuestiones de coordinación y administración de la Universidad, y

- Mejorar la calidad de la docencia y la investigación, a través de un nuevo sistema objetivo y transparente, que garantice el mérito y la capacidad en la selección y el acceso del profesorado.

Por ello, los elementos básicos que se integran en el sistema a distancia, y cuyas características y funciones se diferencian sustancialmente de los de los sistemas convencionales (el alumnado, el docente, los medios o sistemas de comunicación entre ambos, y la estructura organizativa), elementos que deben de ser entendidos en una continua interacción, y no como elementos estancos, se convierten en un instrumento para la consecución de la calidad. 